Article

\title{
Simulations and Subjective Rating of Acoustic Conditions in a Symphony Orchestra-A Case Study
}

\author{
Magne Skålevik 1,2 (D) \\ 1 AKUTEK, 3430 Spikkestad, Norway; msk@brekkestrand.no; Tel.: +47-4147-9375 \\ 2 Brekke \& Strand, 0275 Oslo, Norway
}

Received: 26 May 2019; Accepted: 20 June 2019; Published: 22 July 2019

\begin{abstract}
Acoustic conditions in a symphony orchestra on a concert hall stage are very different from those on an empty stage. Since inter-orchestral sound transmission and other acoustic conditions with the orchestra present is easier to simulate than to measure, a method for simulations in Odeon models of orchestras in different rooms was developed by this author. This method was applied in the Grieghallen Renewal Project, which involved changes in concert hall, orchestra pit, and rehearsal hall. The resident orchestra members gave their overall rating of playing conditions in the home venues in addition to a number of international venues. Acoustical conditions in the rated venues were simulated and compared with ratings. Several metrics were investigated, and their correlation with subjective ratings varied between $r^{2}=0.09$ and $r^{2}=0.85$. It turned out the orchestra clearly preferred to play in conditions where the direct component and the reverberant component of the inter-orchestral sound-transmission on average were equally strong; $|\mathrm{D}-\mathrm{R}|=0$. Any deviation from equality was associated with reduced preference, with correlation coefficient $r=-0.92$. Several interesting implications and interpretations of the result are discussed in the paper.
\end{abstract}

Keywords: acoustics; stage acoustics; orchestra; direct-to-reverberant sound balance; D-R balance; direct sound; reverberation distance

\section{Introduction}

This paper presents a method in which acoustical simulations in 3-D models is used to investigate objective differences that could explain a symphony orchestra's varying preference for different venues. One of the advantages with a 3-D model is that it offers acoustical data with the orchestra present and that any findings can be used in the evaluation of venues that are not yet built, offering support in the design phase.

\subsection{Literature Review and Previous Research in Stage Acoustics}

A review of research in the history of Stage Acoustics was presented by Gade [1] in 2010. Much of the previous work has emphasized the musicians' need to hear oneself and co-players and how to control reflections to provide for such self and mutual hearing. Many room acoustical parameters and quantities have been suggested and tested for their ability to describe the acoustical conditions, but two parameters have so far been included in an ISO-standard, namely $\mathrm{ST}_{\text {early }}$ and $\mathrm{ST}_{\text {late, }}$ initially

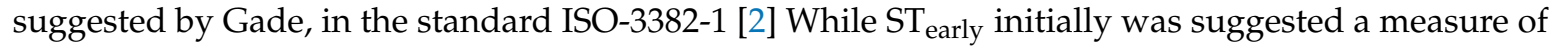
the perceived acoustical support for the individual musician and indeed interpreted and practiced as such, the standard assigns the quantity $\mathrm{ST}_{\text {early }}$ to the aspect of ensemble conditions. $\mathrm{ST}_{\text {late }}$ is in the standard assigned to the aspect of reverberance as perceived by the musician.

While the way the ST-measurements are performed, $1 \mathrm{~m}$ from the source on an empty stage, intuitively makes them an obvious measurement of the room response that a soloist hears when playing 
her/his instrument, it is less intuitive that these measurements have much to do with what a musician on the occupied stage hears when playing in a symphony orchestra.

Inter-orchestral sound transmission as such was measured, e.g., by Krokstad et al. [3] (1980), Halmrast [4] (2001), and Skålevik [5] (2007). Dammerud [6] studied inter-orchestral sound transmission in a scale model. Such investigations have not yet led to a replacement of empty-stage measurements despite evidence of a significant impact from the presence of an orchestra. A typical observation has been the trend of a linear sound level attenuation in the inter-orchestral transmission in contrast to the inverse square law attenuation of direct sound. Moreover, various masking effects due to sound from co-players are expected to play a role.

Since 2010, loudness issues in orchestras have received more attention, and as a part of this, the level balance between sound from a musician's own instrument and the sum of direct and reflected sound from co-musicians. This research is driven by two concerns: the noise-and-health concerns in orchestras; the forward masking by early reflections, which affects the hearing of own instrument and other instruments. In attempts to protect musicians' hearing as well as to improve the perceived balance between individual instruments, groups, and the amount of reverberant sound added to it, it is important to know how strong these components are relative to each other at the musicians' and conductor's ears. Only with this information established, one would know how to make any improvements; for example, to reduce reverberant sound for noise and health reasons would not be adequate if reverberant sound is only a minor part of the sound exposure.

When it comes to quantifying the portions of the aforementioned sound components, the work of Wenmaekers et al. [7] (2015) and the work of Skålevik [8] (2015) may have arrived at different results. While the first concluded that, when playing in an orchestra, sound from the own instrument plays a minor role in the noise exposure, the second concluded that sound from the own instrument accounted for roughly one half the noise exposure.

Of special relevance to this paper, Gade [9] discussed the relationship between loudness, absorption area, reverberation radius, direct-to-reverberant ratios, and other acoustical issues in orchestra rehearsal rooms.

\subsection{Case Background}

Grieghallen (Grieg Memorial Hall) in Bergen, Norway, home of Bergen Philharmonic Orchestra (BPO), was inaugurated in 1978. After 34 years of wear and tear, the Grieghallen Renewal Project started in 2012 and completed in 2015, the year of BPO's 250th anniversaries (BPO is one of the oldest orchestras in the world). The renewal included new seats; a new stage floor; a new orchestra shell; a new and larger orchestra pit in Griegsalen; the 1500-seat main concert hall; and a complete renovation of Peer Gynt, a smaller multipurpose hall which BPO uses for rehearsals now and then. This author was the acoustic consultant of the renewal project, the owner of Grieghallen being the client. For the acoustic consultant, the task was to maintain the acoustic conditions for BPO and audience in Griegsalen, to provide for good acoustic conditions in the new orchestra pit, and to markedly improve the acoustical conditions for BPO during rehearsals in Peer Gynt.

\subsection{Subjective Data}

It was natural to base all requirements and aims on BPO's preferences as to acoustics. To find out what kind of acoustics BPO liked and disliked, a survey among the musicians was carried out. In an online questionnaire, all members of the orchestra were asked to assess the acoustics on stage in Griegsalen, in the orchestra pit in Griegsalen, during rehearsals in Peer Gynt, and in several other concert halls in the world where they have played. The question made clear that they were to assess the performers conditions-not the listeners conditions: "Assess the acoustic conditions for orchestra play in these halls" on a scale from 1 to 5 . Among a total of 50 respondents, it turned out that most of them were able to assess the following venues: Griegsalen stage (GS), Griegsalen Pit (GP), Peer Gynt (PG), Amsterdam Concertgebouw (AC), Vienna Musikverein (VM), Oslo Konserthus (OKH), 
and Boston Symphony Hall (BSH). Since 2004, OKH has had an orchestra canopy and was assessed in its two versions-OKH 1 (before 2004) and OKH 2 (after 2004). We, therefore, consider 8 different venues. Average assessment scores and number off assessors per hall can be seen from diagram and table in Figure 1. In the following, it is described how these scores can be explained by acoustical parameters predicted by simulations in 3-D models of each of the 8 venues.

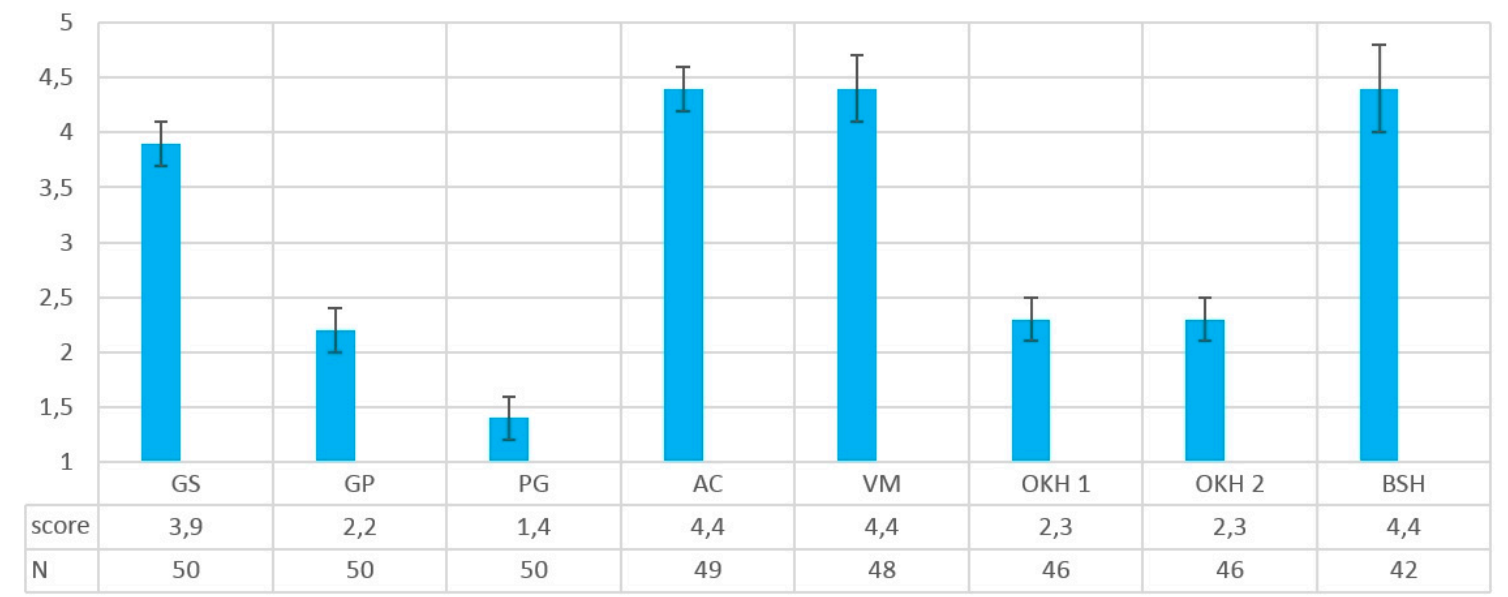

Figure 1. Average assessment scores in 95\% confidence intervals and number of assessors (N).

\subsection{Objective Data—Compact Definitions of Metrics (Parameters)}

Metrics or parameters occurring in this paper are according to textbooks and ISO 3382. A compact set of definitions follows. All single value data are mid-frequency averages of octaves 500 and $1000 \mathrm{~Hz}$.

G $\quad(\mathrm{dB})$

Sound Strength, in decibels, sound pressure level at arbitrary distance from omni-directional source, relative to level of direct sound at 10-m distance from the same source

$G_{\mathrm{d}} \quad(\mathrm{dB}) \quad G$ from the direct sound component only, i.e., $\mathrm{G}$ in anechoic condition

$G_{\mathrm{r}} \quad(\mathrm{dB}) \quad$ Strength of reflected, i.e., reverberant, sound

$G_{\text {late }} \quad(\mathrm{dB}) \quad$ Strength of reflected sound arriving $80 \mathrm{~ms}$ or later after direct sound

$T_{30} \quad$ (s) Reverberation time of a room, calculated from the slope of the decaying sound tail, in the 30- $\mathrm{dB}$ interval between -5 and $-35 \mathrm{~dB}$

ST early

(dB) Stage support, level of reflected sound energy in the 20-100 ms interval, related to energy in the 10-20 ms interval, from an omni-directional source at 1-m distance, average of octave bands 250 through $1000 \mathrm{~Hz}$

$\mathrm{ST}_{\text {late }}(\mathrm{dB}) \quad$ Stage support, level of reflected sound energy in the 100-1000 ms interval, related to energy in the 10-20 ms interval, from an omni-directional source at 1-m distance, average of octave bands 250 through $1000 \mathrm{~Hz}$

D-R (dB) Direct-to-reverberant sound level balance, $D-R=G_{d}-G_{r}$, i.e., $10 \times \lg (d / r)$ where $d / r$ is the ratio between direct and reverberant sound energy 


\section{Method}

\subsection{3-D Models for Simulation}

In search for room acoustical properties that could explain why some of the venues are better liked or less liked by the orchestra members, simulations were performed in 3-D models of each of the 8 venues. For this purpose, the computer software ODEON 11 was used. 2D-renderings of the 3D-models are presented in Supplementary Materials.

To all possible extent, the models were adapted to the actual conditions under which the musicians had assessed the halls, including the occupied stages and audience seating areas in the various concert halls.

The orchestra models were basically a copy of the orchestra model in the GS model but adapted to fit into the constraints and the rake of the various concert hall stages. All orchestra models were plane surfaces of total area in the interval $98-101 \mathrm{~m}^{2}$, with absorption spectrum given by the factory setting "11,000 Orchestra" in Odeon, and scattering coefficient 0.7. In GS, GP, and PG, the absorbing plane is just above the floor plane. In GP (Griegsalen orchestra pit), the seating layout is naturally different from the one on stage and the model was adapted to fit the geometry of the pit prior to 2012. It was assumed that the orchestra would use all available floor, and the resulting area of the orchestra model was $112 \mathrm{~m}^{2}$.

\subsection{Source, Receivers, and Parameters in the Simulations}

All simulated parameters and notations are as defined in Odeon and in accordance with definitions in ISO 3382. In the context of this paper, two of the parameters deserve an explanation. Sound strength $G$ can be decomposed in the direct sound and reflected sound components $G_{d}$ and $G_{r}$, respectively. The balance between the two, $G_{d}-G_{r}$, will be equal to the direct-to-reverberant sound balance D-R, frequently used in many fields of acoustics but less so in concert hall acoustics. While the two G-components are very difficult to decompose from field measurements, they are easy to simulate in ray-based simulation programs like Odeon. In this paper, the equivalents $D-R$ and $G_{d}-G_{r}$ are alternately used depending on context. D-R is used because it emphasizes a conceptual meaning of the balance between direct and reverberant sound that many acousticians will relate to.

In addition to the default parameters calculated by Odeon, we wanted to simulate and measure the inter-orchestral sound transmission. For this purpose, the orchestra was divided into a receiver grid of $1.5 \mathrm{~m} \times 1.5 \mathrm{~m}$ resulting in 43 receivers (in the orchestra pit, this was achieved by a grid of $1.6 \mathrm{~m}$ $\times 1.6 \mathrm{~m}$ at a height of $1.2 \mathrm{~m}$ above the absorbing plane). A source was positioned between the typical positions of a conductor and a concertmaster at a height of $1.0 \mathrm{~m}$ above the absorbing plane. A set of 43 sound strength values $(G)$ from the 43 source-receiver combinations in each venue was acquired, separated into $G_{d}$, i.e., the direct (non-reverberant) part of $G$ and $G_{r}$, i.e., the reverberant part of $G$. The idea was to be able see any explanations of the musicians' preference in not only the total $G$ but also $G_{d}$ or $G_{\mathrm{r}}$ or in the balance between the reverberant and non-reverberant parts, $G_{\mathrm{r}}-G_{\mathrm{d}}$.

Other parameters appearing in this paper are the Reverberation Time $T_{30}$, late sound strength $G_{\text {late, }}$ i.e., strength of sound reflections arriving later than direct sound, and the support parameters $\mathrm{ST}_{\text {early }}$ and $\mathrm{ST}_{\text {late }}$.

\section{Result}

\subsection{Predicted Data-Simulated Room Acoustical Parameters}

Results from the simulated measurements in terms of average of the 43 source receiver combinations are given in Table 1. Parameters are denoted in the heading of each column. " $X$ " is a set of reference values that will be described below. More parameters were calculated, and the selection of the parameters included will be explained further below. The data was used as input in the investigation of potential correlation between the parameters (the predicted data) and the assessment scores 
(the subjective data) presented above. All dB values in the table are rounded to the nearest integer; thus, the column $G_{r}-G_{d}$ is not always equal to the difference between its rounded terms. In the common dichotomy of subjective data and objective data, predicted data are considered here to be the objective data.

Table 1. Results from simulations in the various venue models. " $X$ " is a reference. See text.

\begin{tabular}{ccccccccc}
\hline Venue & $\begin{array}{c}G_{\mathbf{r}}-G_{\mathbf{d}} \\
(\mathbf{d B})\end{array}$ & $\begin{array}{c}G_{\mathbf{r}} \\
(\mathbf{d B})\end{array}$ & $\begin{array}{c}\boldsymbol{T}_{\mathbf{3 0}} \\
(\mathbf{s})\end{array}$ & $\begin{array}{c}G_{\text {late }} \\
(\mathbf{d B})\end{array}$ & $\begin{array}{c}\mathbf{S T}_{\text {late }} \\
(\mathbf{d B})\end{array}$ & $\begin{array}{c}G_{1} \\
(\mathbf{d B})\end{array}$ & $\begin{array}{c}\mathbf{S T}_{\text {early }} \\
(\mathbf{d B})\end{array}$ & $\begin{array}{c}G_{\mathbf{d}} \\
(\mathbf{d B})\end{array}$ \\
\hline GS & 1 & 8 & 1.7 & 2 & -18 & 11 & -15 & 7 \\
\hline GP & 5 & 10 & 1.0 & -4 & -25 & 12 & -14 & 5 \\
\hline PG & 6 & 13 & 1.4 & 9 & -13 & 14 & -12 & 7 \\
\hline AC & -2 & 5 & 2.1 & 1 & -20 & 9 & -19 & 6 \\
\hline VM & 0 & 7 & 2.0 & 2 & -18 & 10 & -12 & 7 \\
\hline OKH 1 & -3 & 4 & 1.5 & 0 & -21 & 9 & -16 & 7 \\
\hline OKH 2 & -3 & 3 & 1.6 & 0 & -21 & 9 & -18 & 7 \\
\hline BS & -1 & 5 & 2.2 & 0 & -22 & 9 & -19 & 6 \\
\hline$X$ & 0 & 7 & 2.2 & 2 & -19 & 10 & -20 & 6 \\
\hline
\end{tabular}

\subsection{Correlation Between Subjective and Predicted Data}

In search for any correlation between the acquired sets of subjective data and predicted data, it was assumed that, for each parameter in Table 1, there exists an unknown optimum value, from which any deviation, positive or negative, would be associated with reduced preference. The more deviation, the less preference. The optimum values in row " $X$ " can be found by running an iteration process for each parameter, e.g., starting with $G$. First, suggest an optimum value $G_{X}$ for $G$. For each venue, calculate how much $G$ deviates from $G_{X}$ in terms of the absolute value $\left|G_{-}-G_{X}\right|$. Then, calculate the correlation between these values and the assessment scores above. Then, suggest another $G_{X}$ and recalculate correlation. Repeat until the highest possible correlation occurs. Example: For the G-parameter, the highest correlation occurred at $G_{X}=10 \mathrm{~dB}$; Since JND of $\mathrm{G}$ is $1 \mathrm{~dB}$, the numbers given in the G-column in Table 2, are simply the absolute value deviations of $\mathrm{G}$ from $10 \mathrm{~dB}$.

Table 2. Absolute deviations (in JND) from the X-values in Table 1 and correlation $\mathrm{r}$ and $r^{2}$ between deviations and score. Tables present $\mathrm{dB}$ and JND values rounded to nearest integer only.

\begin{tabular}{cccccccccc}
\hline Venue & $\begin{array}{c}G_{\mathbf{r}}-G_{\mathbf{d}} \\
(J N D)\end{array}$ & $\begin{array}{c}G_{\mathbf{r}} \\
(J N D)\end{array}$ & $\begin{array}{c}T_{30} \\
(J N D)\end{array}$ & $\begin{array}{c}G_{\text {late }} \\
(J N D)\end{array}$ & $\begin{array}{c}\text { ST }_{\text {late }} \\
(\text { JND) }\end{array}$ & $\begin{array}{c}G \\
\text { (JND) }\end{array}$ & $\begin{array}{c}\text { ST }_{\text {early }} \\
\text { (JND) }\end{array}$ & $\begin{array}{c}G_{\mathbf{d}} \\
\text { (JND) }\end{array}$ & Score \\
\hline GS & 1 & 1 & 4 & 0 & 1 & 1 & 5 & 1 & 3.9 \\
\hline GP & 5 & 3 & 11 & 6 & 6 & 2 & 6 & 1 & 2.2 \\
\hline PG & 6 & 6 & 7 & 7 & 6 & 4 & 8 & 1 & 1.4 \\
\hline AC & 2 & 2 & 1 & 1 & 1 & 1 & 1 & 0 & 4.4 \\
\hline VM & 0 & 0 & 2 & 0 & 1 & 0 & 8 & 1 & 4.4 \\
\hline OKH 1 & 3 & 3 & 6 & 2 & 2 & 1 & 5 & 1 & 2.3 \\
\hline OKH 2 & 3 & 3 & 5 & 2 & 2 & 1 & 2 & 1 & 2.3 \\
\hline BSH & 1 & 1 & 0 & 2 & 3 & 1 & 1 & 0 & 4.4 \\
\hline$r^{2}$ & 0.85 & 0.74 & 0.71 & 0.64 & 0.51 & 0.49 & 0.22 & 0.09 & 1.0 \\
\hline$r$ & -0.92 & -0.86 & -0.84 & -0.8 & -0.71 & -0.7 & -0.47 & -0.29 & -1.0 \\
\hline
\end{tabular}


Then, do the above iteration process for all parameters, one column at the time, in arbitrary sequence. Finally, calculate the deviations from optimum, i.e., the differences between each value in Table 1 and its optimum value in row " $X$ ". In Table 2, the deviations are expressed in terms of Just Noticeable Difference (JND), assuming $1 \mathrm{~dB}$ is $1 \mathrm{JND}$, and the $5 \%$ difference in $T_{30}$ is $1 \mathrm{JND}$. The rows $r$ and $r^{2}$ are the correlation and the squared (Pearson) correlation between the JND's in a parameter column and the Score column. Calculations are made with several decimals, but for reader friendliness, the tables show integer values only of $\mathrm{dB}$ and JND.

Comment on $G_{\mathrm{d}}$ : In the background data, the $G_{\mathrm{d}}$ parameter values varies between $5.3 \mathrm{~dB}(\mathrm{GP})$ and $7.2 \mathrm{~dB}$ (GS). The low value in GP occurred in the orchestra pit. The other venues are statistically $6.8 \pm 0.3 \mathrm{~dB}$, partly due to variations in layout and partly due to random variations in the receiver positions generated automatically in Odeon. Note: The average of $G_{r}-G_{d}$ in each orchestra grid should be used, instead of average of $G_{r}$ minus average of $G_{d}$. The former is the parameter representing the perceived aspect. The latter would in general yield different results.

\subsection{First Sight Comments to Correlation Results}

The parameters are presented in descending order of $r^{2}$ toward the right. All default parameters in Odeon were tested, but only those of special interest (traditional podium parameters) or those with higher $r^{2}$ correlation, and thereby the better explanation potential, are included in Table 2. Surprisingly, the parameter " $G_{\mathrm{r}}-G_{\mathrm{d}}$ " turned out to be the one that best explained the musicians' assessment of the various venues, with $r=-0.92$ and $r^{2}=0.85$, as graphically illustrated by the plot in Figure 2. The spread around the dashed regression line indicates an uncertainty of around $\pm 1 \mathrm{JND}$, i.e., $\pm 1 \mathrm{~dB}$.

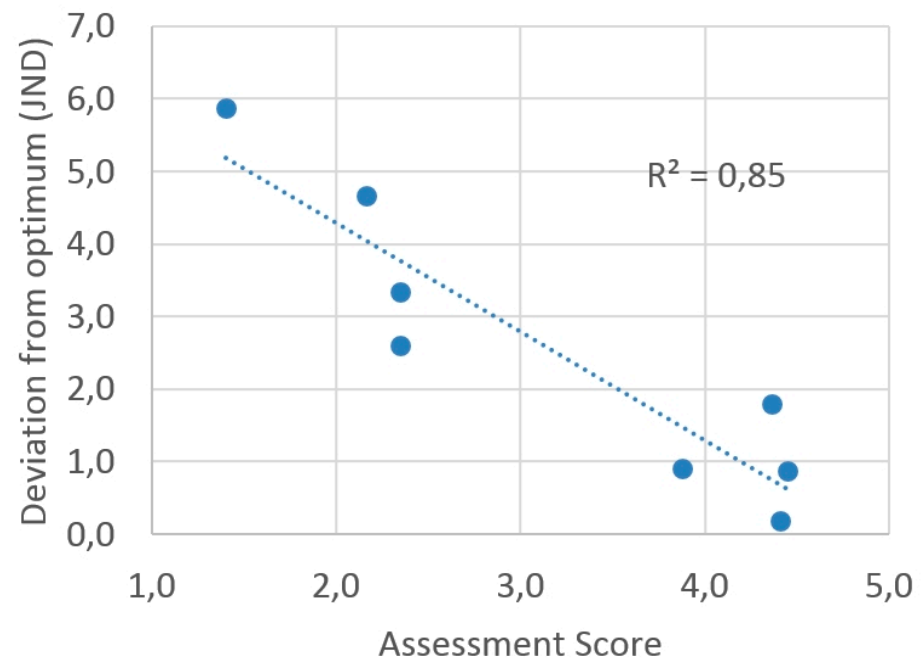

Figure 2. Plot of subjective score (horizontal axis) versus the absolute deviation of $\left|G_{r}-G_{d}\right|$ from its optimum value. See Table 2 for numeric values.

We interpret the results as follows: When $G_{r}-G_{d}$ is approximately equal to zero, i.e., the reverberant and direct sound transmission level between the concertmaster/conductor and other positions in the orchestra are on average equal, when simulated in an Odeon model, the BPO orchestra musicians are likely to judge the acoustic conditions for orchestra play as "very good".

Reverberant sound transmission $G_{r}$ alone correlates considerably with score, $r^{2}=0.74$, and its variation is the main source of variation in " $G_{\mathrm{r}}-G_{\mathrm{d}}$ ". However, though the information in $G_{\mathrm{d}}$ brings no useful explanation alone $\left(r^{2}=0.09\right)$, it contributes to improved explanation (from 0.74-0.85) when combined with $G_{\mathrm{r}}$ because of conditions in the orchestra pit. Reverberant transmission in the orchestra pit, $G_{\mathrm{r}}=10 \mathrm{~dB}$, deviates by $3 \mathrm{~dB}$ from optimum, which is not enough to explain the musicians' assessment of the acoustic conditions there. Loudness in the pit also does not appear to be extraordinary ( $G$ is only $1 \mathrm{~dB}$ higher in GP than GS). However, the direct sound is, on average, weaker than elsewhere 
because of the elongated layout in the pit (thus longer inter-orchestral distances), resulting in a very high reverberant-to-direct balance, $\left|G_{\mathrm{r}}-G_{\mathrm{d}}\right|=5 \mathrm{~dB}$, which deviates $5 \mathrm{JND}$ from the optimum. In such conditions, musicians hear each other mostly through reverberant sound and relatively little direct sound is heard there.

As a preliminary conclusion, the musicians in BPO prefer a balance between reverberant sound and direct sound, on average $G_{\mathrm{r}}-G_{\mathrm{d}}=0$. This corresponds to an average Direct-Reverberant level balance D-R equal to $0 \mathrm{~dB}$.

Note: It is important to keep in mind that the findings above are valid to simulations in 3-D models like those described above. This make the results relevant in design tasks, since simulations are available, and often the only available data in the design phase. In further work, it would be interesting to investigate to what degree the findings would translate to field measurements.

\section{Discussion}

It is important to bear in mind that the above is a case study and that the assessments may be biased by the reputation of MV, AC, and BSH. Moreover, other orchestras may have other preferences. On the other hand, its results and findings have some interesting implications and represent a starting point for further research. It would be interesting to apply the same approach in other orchestras and projects to test if similar results can be found.

Apart from presenting and discussing the results of a case, this paper presents a method that could support designers of new or refurbished rehearsal spaces or performance spaces for a resident symphony orchestra. In this respect, the results and implications suggest that the D-R balance should be included among the parameters under consideration.

\subsection{Direct-to-Reverberant Ratio and Balance}

A common parameter in audio engineering is the direct-to-reverberant ratio $\mathrm{d} / \mathrm{r}$ and the direct-to-reverberant level $\mathrm{D}-\mathrm{R}=10 \times \log (\mathrm{d} / \mathrm{r})$. $\mathrm{D}-\mathrm{R}$ is a measure of the level balance between the direct and the reverberant component in sound transmission or in a transfer function. A sound field in the neighborhood of a sound source, where $\mathrm{D}-\mathrm{R}>0$, is dominated by direct sound, hence referred to as the direct sound region. Gade (2012) mentions this in the context of mutual hearing in orchestras [9]. The distance from a sound source at which the direct and reverberant sound is equally strong, $\mathrm{D}=\mathrm{R}$, is defined the critical distance $d_{c}$. At longer distances, sound transmission is dominated by reverberant sound, $\mathrm{R}>\mathrm{D}$, i.e., in the reverberant sound field. In the case of an omni-directional source, $d_{c}=r_{r}$, where $r_{r}$ is the reverberant radius or the hall radius, a property of the room,

$$
r_{r}=0.57 \times(\mathrm{V} / \mathrm{T})^{0.5}=0.14 \times \mathrm{A}^{0.5}
$$

where $V$ is room volume, $T$ is reverberation time, and $A$ is absorption area. This assumes classical diffuse conditions. In the case of stronger or weaker early reflections, $d_{c}$ and $r_{r}$ would be shorter or longer, respectively. The above description is the conventional way to interpret the reverberant radius and the direct and reverberant sound fields, i.e., from the source' perspective. Figure 3 is a plot of D-R plotted against distance from the concertmaster to all occupied positions, distributed over the orchestra and audience in the GS model. To the average orchestra member, the distance is $4.9 \mathrm{~m}$ and $D-R$ is $0.2 \mathrm{~dB}$. To the average audience member, the distance is $25 \mathrm{~m}$ and $\mathrm{D}-\mathrm{R}$ is $-8.5 \mathrm{~dB}$.

However, for the individual musician in a symphony orchestra, it may be more relevant to take the receiver's perspective as follows. Draw a circle with radius equal to $r_{r}$, around a musician or a conductor. From the conductor's perspective, musicians' can be divided in two categories- the near group, i.e., those inside the circle, and the far group, i.e., those outside the circle (Figure 4, right). The sound from the near group will be mainly direct sound, and the sound from the far group is mainly reverberant sound. A visual analogy to the reverberant radius would be sight range in navigation, road traffic, and aviation, which is a measure of how far one can see clearly. Reverberant sound would be an 
analogy to fog and mist, which, in a similar manner, makes it harder to separate details. Transparency, another attribute used in seeing as well as in hearing, would increase as D-R increases.
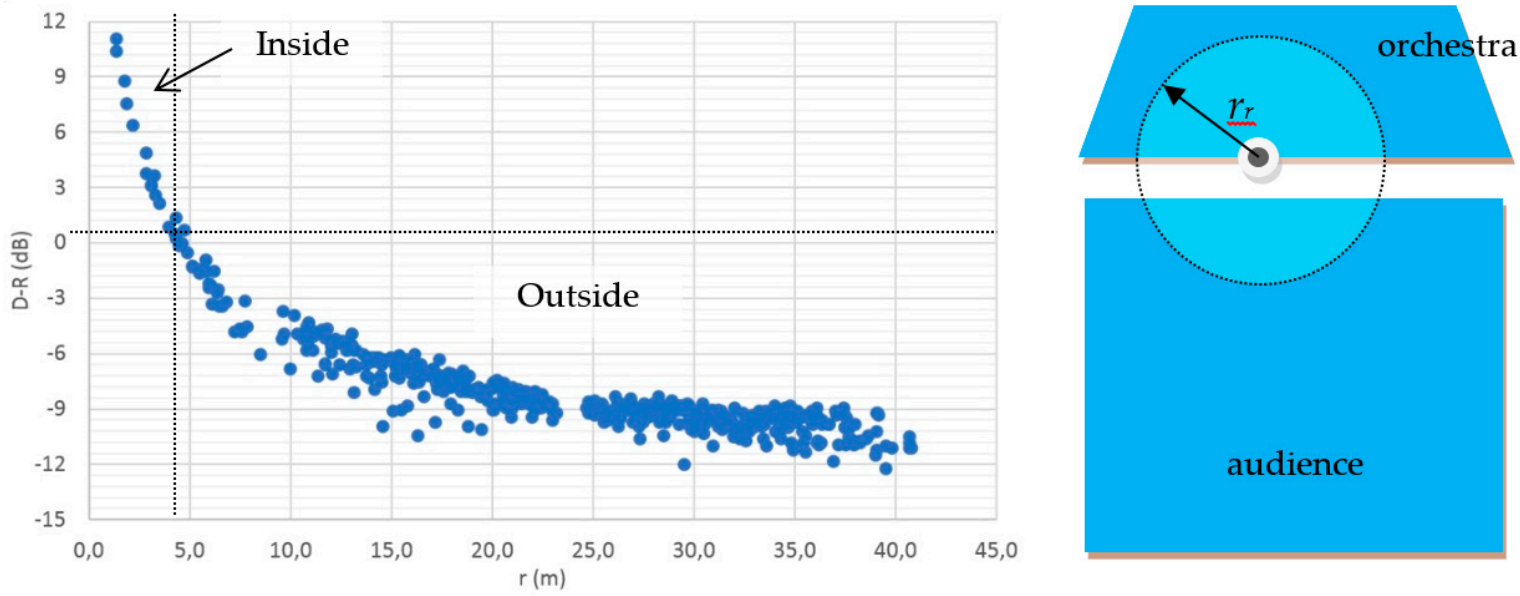

Figure 3. Direct-to-reverberant sound balance (D-R) plotted against distance from the concertmaster to all occupied positions distributed over the orchestra and audience in the GS model. Right: Schematic plan, source (dot), dotted circle line with radius $r_{r}$ (arrow) $=4.5 \mathrm{~m}$. Inside and outside of the circle corresponds to quadrants in the plot to the left.
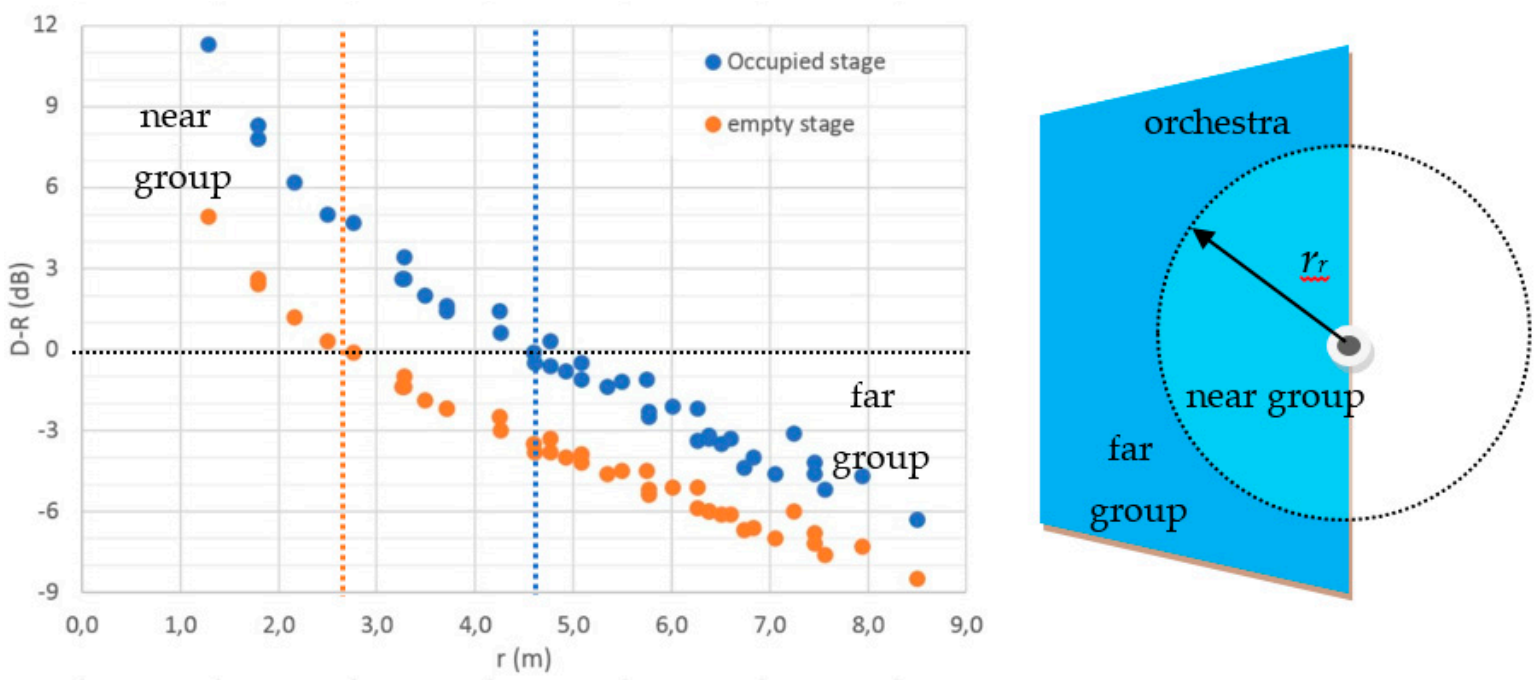

Figure 4. Direct-to-Reverberant level (dB) measured from simulations in the 3-D model of GS. See text.

Extending from hearing to inter-orchestral communication: A musician communicates via direct sound with the near group and via reverberant sound with the far group. Changes in the D-R balance would mean changes to the near and far groups and the balance between them. It is hypothesized that musicians' and the conductor are sensitive to such changes and that D-R balance is a critical aspect in stage acoustics for symphony orchestras. When an orchestra is on tour, visiting one concert hall after the other, acoustic conditions change more or less from one day to the other. Likewise, $r_{r}$, varies from venue to venue, i.e., if the ratio $\mathrm{V} / \mathrm{T}$ varies. If the orchestra members prefer a certain $\mathrm{D}-\mathrm{R}$ balance, a change in $r_{r}$ may be unfortunate or challenging and difficult to adapt to without ideals being compromised.

In Figure 4, simulated midfrequency (500 and $1 \mathrm{k}$ octave) $\mathrm{D}-\mathrm{R}$ balance levels in $\mathrm{dB}$ are plotted against distance between the concertmaster/conductor and 43 positions distributed in a $1.5 \mathrm{~m}$ grid over the orchestra in the GS model. The average is $\mathrm{D}-\mathrm{R}=0.1 \mathrm{~dB}$, and the reverberant radius appears to be $r_{r}$ 
$=4.5 \mathrm{~m}$. The near group is in the upper left quadrant and inside the circle. For simulated measurements over the same points over the empty stage, the average is $\mathrm{D}-\mathrm{R}=-3.4 \mathrm{~dB}$ and the reverberant radius is around $2.5 \mathrm{~m}$.

Note that D-R is, on average, $3.5 \mathrm{~dB}$ higher without the orchestra (empty stage floor) than over the orchestra. Moreover, the difference between an empty and occupied stage is bigger at short distances and smaller, mainly due to early reflected sound being more effectively absorbed at short distances, at long distances in the model. These differences indicate that D-R measurements on an empty stage may not be useful when trying to understand the conditions for inter-orchestral hearing. Since measurements with orchestra present would be very time- and resource-consuming, simulations will be the only practical option. Moreover, during planning of a new concert hall or changes to an existing one, only simulations are available. We need is a reliable criteria for the simulated measurements. It would require more investigations to settle whether there exists a global criterion or if there are local criteria due to varying preference in orchestras. Until further we suggest that the criterion is orchestra specific. In a research phase, real measurements would be performed to verify simulations.

Music instruments, in general, can have complex directivity patterns, generally more directive toward higher frequencies. Directivity of harmonics fluctuate with time in a given direction and fluctuate over directions at a given instant. Long-time average directivity patterns can be established from measurements, but fluctuations around the average can, in $2 / 3$ of the time, be more than $\pm 5 \mathrm{~dB}$ in a violin and more than $4 \mathrm{~dB}$ in an oboe [10]. Importantly, the $\mathrm{D}-\mathrm{R}$ can, in $1 / 3$ of the time, be more than $5 \mathrm{~dB}$ above the long-term $\mathrm{D}-\mathrm{R}$ of the source. This is one of the reasons why an instrument can be localized at distances far beyond $d_{c}$, by analogy to the visual effect known as glimpsing.

The other reason is binaural hearing, i.e., our ability to combine information at our two ears and, in particular, our ability to optimize the inter-aural cross-correlation between sounds at our left and right ears by turning our heads straight toward the source. According to Jeffress' binaural hearing model [11], sounds arriving from different azimuth angles can be separated by our hearing, even in ambient noise and other complex acoustic environments if D-R is above some threshold. An example is the ability to separate single voices in a noisy cocktail party, hence often referred to as the cocktail party effect. Acoustically, a symphony orchestra has common features with a cocktail party where speaking voices have been replaced with playing instruments. For one, there are similar challenges with hearing. Moreover, hearing problems are often in a feedback loop with escalating sound levels, another characteristic feature of cocktail parties, as will be commented further in Section 5.2.

Note: It should be noted that the formulae for quantifying the reverberation distance in terms of $V, T$, and $A$, like in Equation (1), assume diffuse reverberant sound field conditions, and free-field propagation of direct sound. In practice, the sound field close to a symphony orchestra on a concert hall stage is not diffuse in the sense assumed in theory. For example, the orchestra would, to a high degree, obstruct the floor reflection, thus reducing the reverberant component. Therefore, the actual critical distance where $\mathrm{D}-\mathrm{R}=0$ would be longer than the theoretical one, as demonstrated in the difference between an empty stage and an occupied stage in Figure 4. Moreover, two venues with equal $V / T$ can have different $G_{r}$, and $G_{r}$ would vary more or less over the orchestra. Finally, direct sound does not propagate through the orchestra in a simple manner like in free-field.

\subsection{Interpretation of BPO's Preference}

As described above, the immediate interpretation of the results is that the orchestra in this case study prefer a balance between reverberant sound and direct sound, $G_{r}-G_{d}=0$, which is equivalent to the average D-R balance being zero. Further, in view of Figure 4, the balance between direct and reverberant sound translates to a balance between the Near Group and the Far Group, or the Near-Far balance for short. It can be seen in the "empty stage" simulations that with a stronger reverberant sound, the D-R balance is lower, and the Near Group is smaller. Thus, if the D-R balance is lower than preferred, the Near Group will be smaller than preferred, and vice versa. See Figure 5. 
A smaller Near Group would mean that fewer musicians hear each other via direct sound and that more musicians hear each other through reverberant sound. For an individual, the count of co-players inside the Near Group would depend on the density of musicians around an individual musician. Typically, there is a higher density in front of an orchestra than at the back. The conductor/concertmaster would have a higher instrument count in their Near Group than a percussionist in the rear row would have: typically 50 string players in the Near Group around the conductor and 30 others (woodwinds and brass percussion) in the Far Group. However, this does not change the fact that in any position, the Near Group would shrink if D-R decreases, and vice versa.

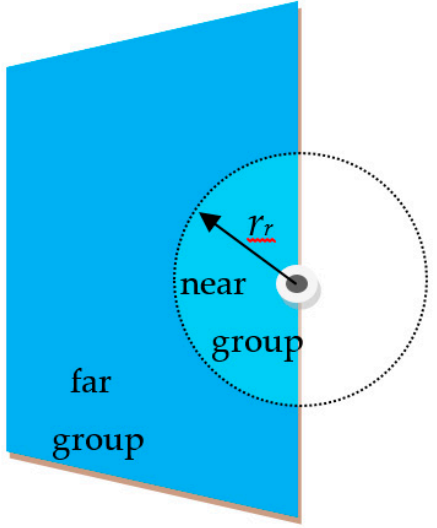

D-R too low

Near Group too small

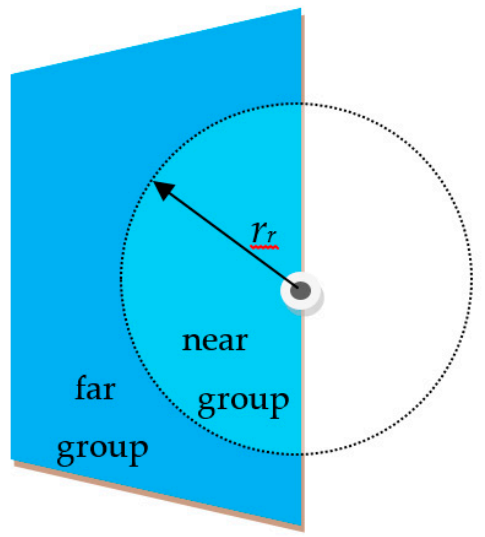

D-R and Near-Far balances as preferred

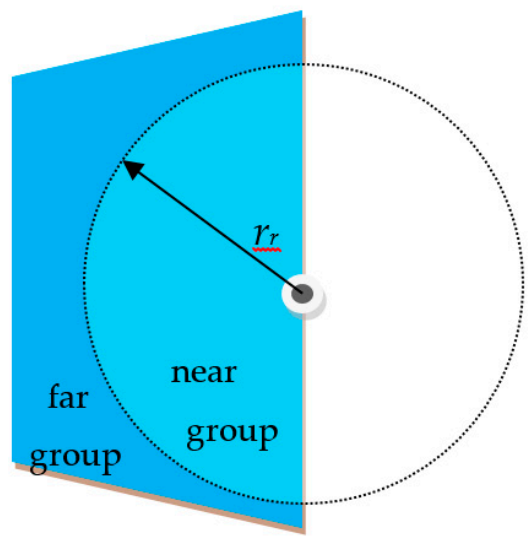

D-R too high

Near Group too big

Figure 5. Interpretation of results in this case study in terms of preferred $r_{r}$ and Near-Far balance.

\section{Outlooking}

\subsection{Two Sides of the Same Coin}

Surprising as it may be, the noise-and-health concern and the mutual hearing concern in symphony orchestras may well be two sides of the same coin. Several authors have suggested that musicians would play stronger in an environment where they hear too little of their own instrument. If reflected sound is a significant portion of the sound exposure, unfortunate sound reflections may, due to their masking effect, lead to forced playing. Consequently, such unfortunate reflections may drive a vicious circle, driving the whole orchestra to play stronger, making both noise exposure and performance problems even worse because of increased spectral masking and more harsh sounds (forced playing would increase the strength of the upper harmonics more than the lower ones).

\subsection{Understanding Self-Reinforcing Effects and the Escalating Levels Problem}

Similar problems are well-known from cocktail parties, as mentioned above, where escalating hearing problems and noise exposure are indeed two sides of the same coin. The driving factors in the positive feedback loop (raised voices causes even more raised voices, and so on) are the Lombard effect, a biological reflex, and the intuitive effect, i.e., the attempt to overcome hearing problems and to be heard by raising the voice. Moreover, the conductor may demand more sound from instruments or groups if they are not heard well, even if the cause of the problem is the others playing too strong. It is practically impossible to avoid these problems if the reverberant radius is small compared to the distance between individuals in a group that try to communicate, i.e., if trying to communicate with individuals outside the Near Group. To this authors knowledge, the similarities between cocktail parties and symphony orchestras have not been studied by other authors.

Another self-reinforcing effect can occur if sound reflections are too weak, since this could make many orchestra members compensate by playing stronger or to be demanded by the conductor to do 
so. If bass reflections are weak, i.e., low frequency $\mathrm{G}$ is relatively weak, musicians and conductor could perceive a lack of warmth and too much brilliance in the sound. Moreover, the important temporal que in the fundamental of lower pitched instruments would be weak. In this case, the conductor may want more power from the lower pitch instruments. However, in musical instruments, more power cannot be produced without, at the same time, increasing brilliance and reducing warmth. Again, this makes the initial problem worse, driving the same vicious circle as described above. Concludingly, too much or too little reflections both lead to double trouble, i.e., noise and health problems and ensemble problems of hearing oneself and others.

\subsection{Further Work}

In further work, two parallel activities are suggested: (1) The investigation presented in this paper should be extended to more cases, with more orchestras and more venues. (2) Methods for field measurements of the D-R balance in orchestras should be explored, e.g., by field measurements of actual inter-orchestral sound transmission.

\section{Conclusions}

In terms of inter-orchestral D-R as discussed in this paper, too low or too high D-R values would involve the risk of escalating loudness and sound level exposure as well as worsened conditions for inter-orchestral hearing. As a preliminary recommendation, simulated average D-R over the orchestra in a 3-D model with a source in the position of the conductor/concertmaster should be close to zero. Until further it is recommended to include simulated D-R in research or design concerning orchestra acoustics. However, the preference criterion should be considered orchestra specific, until any global criterion can be verified.

Supplementary Materials: The 2D-renderings of the 3D-models are available online at http://www.mdpi.com/ 2624-599X/1/3/33/s1.

Conflicts of Interest: The author declares no conflict of interest.

\section{References}

1. Gade, A.C. Acoustics for Symphony Orchestras; Status After Three Decades of Experimental Research. In Proceedings of the International Symposium on Room Acoustics (ISRA 2010), Melbourne, Australia, 29-31 August 2010; Available online: http://www.akutek.info/Papers/AG_Stage_Acoustics.pdf (accessed on 31 August 2010).

2. International Standard. ISO-3382 Acoustics-Measurement of Room Acoustic Parameters_Part 1, Performance Spaces, 1st ed.; International Organization for Standardization, Chemin de Blandonnet 8, CP 401 - 1214 Vernier: Geneva, Switzerland, 2009.

3. Krokstad, A.; Vindspoll, J.; Sæther, R. Orkesterpodium, Samspill og Solo (Orchestra Platform, Ensemble and Solo); Technical Report; The Laboratory of Acoustics, The Technical University of Trondheim: Trondheim, Norway, 1980.

4. Halmrast, T. Acoustical Society of America, Chicago 4th June 2001, Session: “The First 80 Milliseconds in Auditoria". Available online: http://www.akutek.info/Papers/TH_Coloration2001.pdf (accessed on 13 March 2007).

5. Skålevik, M. Sound Transmission between Musicians in a Symphony Orchestra on a Concert Hall Stage. 2007. Available online: http://www.akutek.info/Papers/MS_Sound_Transmission.pdf (accessed on 17 May 2007).

6. Dammerud, J.J. Stage Acoustics for Symphony Orchestras in Concert Halls. Chapter 4. Available online: http://www.akutek.info/Papers/JJD_Stage_acoustics_PhDthesis_96dpi.pdf (accessed on 20 May 2010).

7. Wenmaekers, R.H.C.; Hak, C.C.J.M. Noise Exposure of Musicians: The Own Instrument's Sound Compared to the Sound from Others (Euronoise, Maastricht 2015). Available online: http://www.akutek.info/Papers/ RW_Own_Instrument_reOthers.pdf (accessed on 8 June 2015). 
8. Skålevik, M. Level Balance between Self, Others and Reverb, and Its Significance to Noise Exposure as Well as Mutual Hearing in Orchestra Musicians, (Euronoise, Maastricht 2015). Available online: http://www.akutek.info/Papers/MS_Self_Others_Reverb.pdf (accessed on 5 June 2015).

9. Gade, A.C. Sound levels in rehearsal and medium sized concert halls; are they too loud for the musicians? In Proceedings of the Meetings on Acoustics, Hong Kong, China, 13-18 May 2012.

10. Directivity of Musical Instruments. Available online: http://www.akutek.info/articles_files/dynamic_ directivity.htm (accessed on 9 December 2011).

11. Jeffress, L.A. A place theory of sound localization. J. Comp. Physiol. Psychol. 1948, 41, 35-39. [CrossRef]

(C) 2019 by the author. Licensee MDPI, Basel, Switzerland. This article is an open access article distributed under the terms and conditions of the Creative Commons Attribution

(CC BY) license (http://creativecommons.org/licenses/by/4.0/). 